\title{
Resonant Plasmonic Enhancement of InGaN/GaN LED using Periodically Structured Ag Nanodisks
}

Fadil, Ahmed; lida, Daisuke; Zhu, Xiaolong; Ou, Yiyu; Chen, Yuntian; Dam-Hansen, Carsten; Petersen, Paul Michael; Ou, Haiyan

Published in:

ACP Technical Digest

Publication date:

2013

Link back to DTU Orbit

Citation (APA):

Fadil, A., lida, D., Zhu, X., Ou, Y., Chen, Y., Dam-Hansen, C., Petersen, P. M., \& Ou, H. (2013). Resonant Plasmonic Enhancement of InGaN/GaN LED using Periodically Structured Ag Nanodisks. In ACP Technical Digest Optical Society of America.

\section{General rights}

Copyright and moral rights for the publications made accessible in the public portal are retained by the authors and/or other copyright owners and it is a condition of accessing publications that users recognise and abide by the legal requirements associated with these rights.

- Users may download and print one copy of any publication from the public portal for the purpose of private study or research.

- You may not further distribute the material or use it for any profit-making activity or commercial gain

- You may freely distribute the URL identifying the publication in the public portal 


\title{
Resonant Plasmonic Enhancement of InGaN/GaN LED using Periodically Structured Ag Nanodisks
}

\author{
Ahmed Fadil ${ }^{1, *}$, Daisuke Iida ${ }^{2}$, Xiaolong Zhu ${ }^{1}$, Yiyu Ou ${ }^{1}$, Yuntian Chen ${ }^{1}$, Carsten Dam-Hansen ${ }^{1}$, \\ Paul Michael Petersen ${ }^{1}$ and Haiyan Ou ${ }^{1}$ \\ ${ }^{1}$ Department of Photonics Engineering, Technical University of Denmark, Ørsteds Plads 343, 2800 Kgs. Lyngby, Denmark \\ ${ }^{2}$ Department of Materials Science and Engineering, Meijo University, 1-501 Shiogamaguchi, Nagoya 468-8502, Japan \\ *afad@fotonik.dtu.dk
}

\begin{abstract}
Ag nanodisks are fabricated on GaN-based LED to enhance emission efficiency. Nanosphere lithography is used to obtain a periodic nano-structure, and a photoluminescence enhancement of 2.7 is reported with $\mathrm{Ag}$ nanodisk diameter of $330 \mathrm{~nm}$.

OCIS codes: (230.3670) Light-emitting diodes; (240.6680) Surface plasmons
\end{abstract}

\section{Introduction}

GaN-based light-emitting diodes (LEDs) have shown a promising role in the search for energy efficient visible light sources. By incorporating indium (In), the bandgap of InGaN can be tuned from near UV to over the entire visible spectrum. LEDs based on III-arsenide and phosphide material systems already constitute efficient light sources from the red to yellow spectral region, thus III-nitrides could play a crucial role for LEDs from blue to green. The internal quantum efficiency (IQE) of blue InGaN/GaN LED grown by metalorganic chemical vapor deposition (MOCVD) on sapphire substrate, easily reaches above $80 \%$. However, in moving towards longer wavelengths by increasing the In composition, the IQE suffers a significant decrease, resulting in a poor performance of green LEDs [1].

One approach to improve the IQE of LEDs, which has been heavily researched over the last couple of years, involves the fabrication of Ag nanoparticles (NPs) in the near field of the active light emitting region, where the coupling between excitons and localized surface plasmon (LSP) modes is expected to provide a faster radiative decay channel [2]. The simplest fabrication approach is self-assembled Ag NPs by thermal annealing [3]. However, because of the difficulty in controlling the particle sizes, this method allows limited control over the LSP resonance.

Here we propose a simple fabrication method of Ag nanodisks (NDs) with a periodic pattern based on nanosphere lithography (NSL), with which both the particle size and the periodicity can be controlled. While the resonance position can be tuned with the size, the periodicity allows control over the resonance width [4].

\section{Fabrication Process}

All the samples were prepared on a multi-quantum well (MQW) InGaN/GaN green LED grown by MOCVD on a $c$ plane sapphire substrate. In order to have QW-LSP coupling the thickness of the Mg-doped p-GaN layer was $27 \mathrm{~nm}$. The process flow of fabricating a periodic structure of Ag NDs using NSL is illustrated in Fig. 1. First two thin layers of $\mathrm{Si}_{3} \mathrm{~N}_{4}$ and $\mathrm{SiO}_{2}$, for pattern reversal, were deposited by plasma enhanced chemical vapor deposition (PECVD), where $\mathrm{SiO}_{2}$ provides an adhesion layer for the dielectric nanospheres and $\mathrm{Si}_{3} \mathrm{~N}_{4}$ is a passivation layer for $\mathrm{GaN}$ surface before $\mathrm{SiO}_{2}$ deposition.

Polystyrene nanospheres with a diameter of $595 \pm 5 \mathrm{~nm}$ were deposited on the surface via the Langmuir-Blodgett technique to form a colloidal structure of NPs, Fig. 1(a). The success of this step was very dependent on temperature, humidity, applied pressure, and the aqueous solution concentration of the nanospheres.

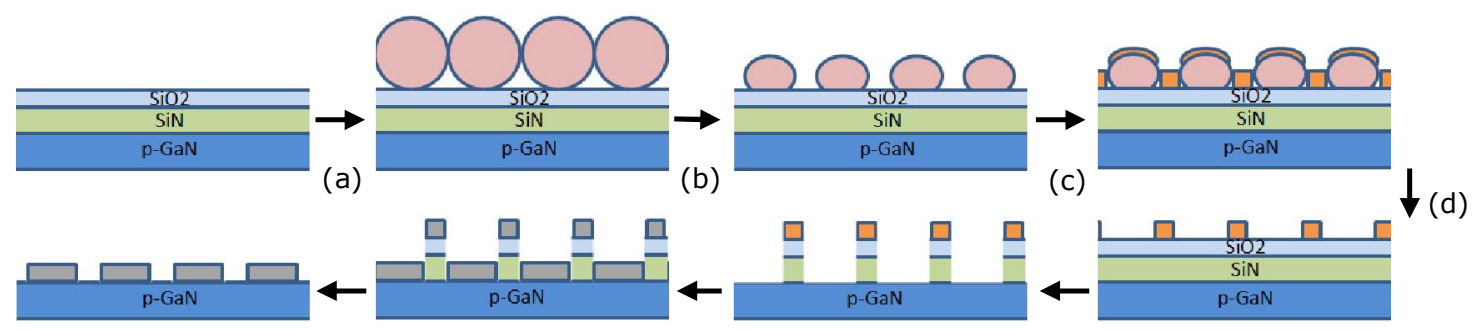

(g)

(f)

(e)

Figure 1: Process flow. 
The diameter of these spheres determines the periodicity (pitch) of Ag NDs. However, to control the size, an $\mathrm{O}_{2}$ reactive ion-etching (RIE) process was employed to reduce the size of the polystyrene nanospheres, Fig. 1(b). The corresponding scanning electron microscope (SEM) image after $\mathrm{O}_{2}$ RIE is shown in Fig. 2(a).

As an etching-mask, a $30 \mathrm{~nm}$ layer of $\mathrm{Ni}$ was deposited by e-beam evaporation, Fig. 1(c), whereafter the nanospheres were removed by sonication in acetone, Fig. 1(d). The pattern formed by the nanospheres was transferred to the $\mathrm{Si}_{3} \mathrm{~N}_{4} / \mathrm{SiO}_{2}$ layer by an RIE process, Fig. 1(e), and the Ni layer was then removed by an aqua regia solution. A $30 \mathrm{~nm}$ layer of $\mathrm{Ag}$ was deposited, Fig. 1(f), and a lift-off process was done through wet etching the $\mathrm{Si}_{3} \mathrm{~N}_{4} / \mathrm{SiO}_{2}$ with $\mathrm{BHF}$, leaving behind a periodic pattern of $\mathrm{Ag}$ corresponding to that of the etched nanosphere pattern, Fig. 1(g). The Ag NPs, however, had the shape of a disk with a diameter equal to that of the etched nanospheres, as shown in Fig. 2(b), and a thickness of $30 \mathrm{~nm}$.
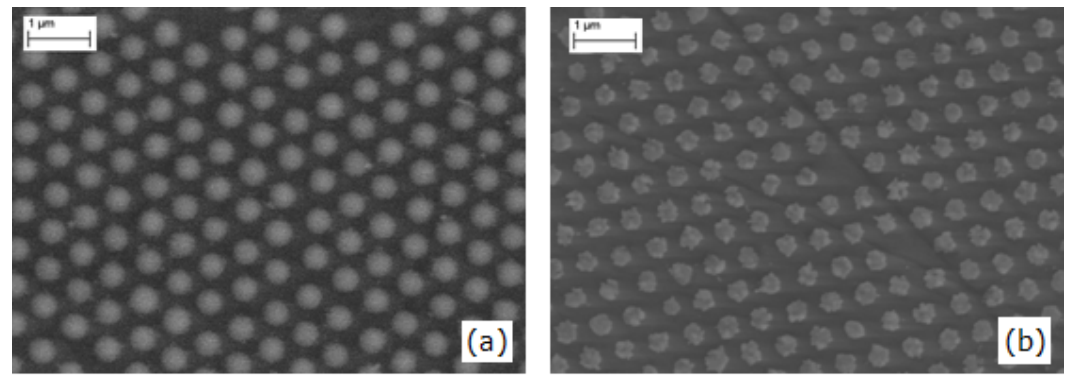

Figure 2: SEM image of (a) reduced-size colloidal nanospheres, and (b) the resulting Ag ND formation.

\section{Optical Characterizations}

Four samples were prepared with different Ag ND average diameters, corresponding to 380, 330, 240 and $130 \mathrm{~nm}$. The photoluminescence (PL) spectra were measured using a backside excitation and detection setup. The excitation laser had a wavelength of $375 \mathrm{~nm}$ and an excitation power of $1 \mathrm{~mW}$. PL spectra of the 380 and $330 \mathrm{~nm}$ samples are shown in Fig. 3(a). The difference in the as-grown PL intensity and peak wavelength is due to variations of In composition across the wafer. Therefore, reference is always taken along contours of equal wavelength. The peak around $425 \mathrm{~nm}$ is from the InGaN/GaN super-lattice (SL) structure of the LED which is grown before the MQWs. It is noticed that the intensity at $425 \mathrm{~nm}$ is almost unaffected by the Ag NDs for both samples, which is due to large spacing between $\mathrm{p}-\mathrm{GaN}$ surface and the SL structure, $\sim 100 \mathrm{~nm}$, leading to an inefficient coupling from excitons to LSP mode at the blue wavelength. This would indicate that the enhanced intensity at the green wavelength is due to MQW-LSP coupling which provides a faster radiative decay channel for the excitons and increases the IQE. Although the as-grown PL spectra for $380 \mathrm{~nm}$ and $330 \mathrm{~nm}$ samples are different, they are same for the 330, 240 and $130 \mathrm{~nm}$ samples as shown in the inset of Fig. 3(a).
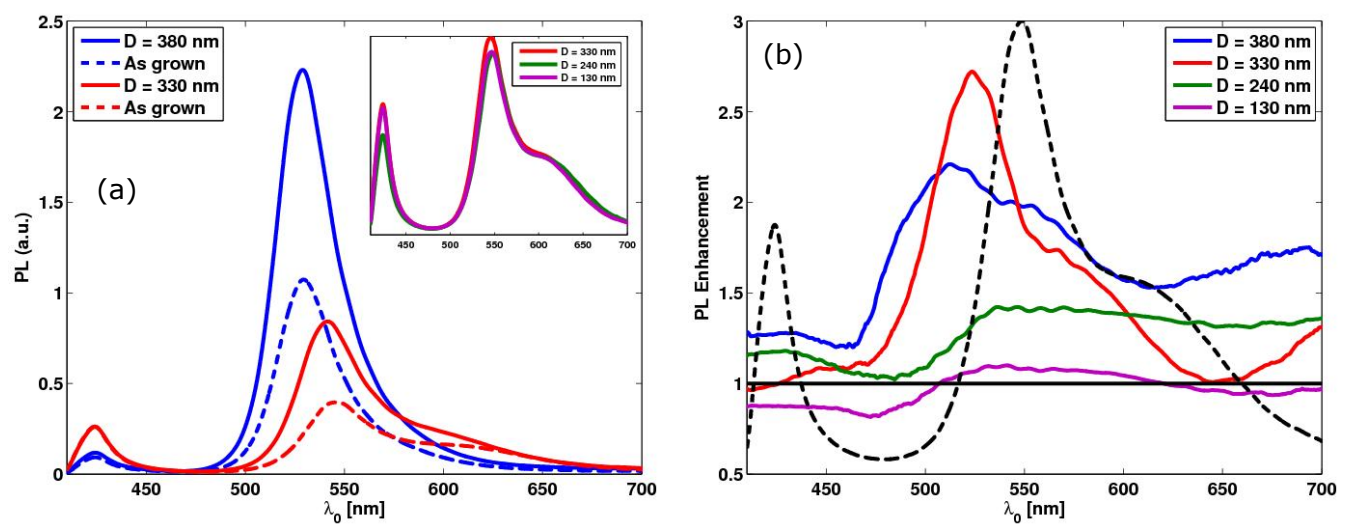

Figure 3: (a) The PL spectra of two samples, comparing areas with (solid) and without (dashed) Ag nanodisks. The inset shows the remaining asgrown PL spectra. (b) PL enhancement factors for samples with different Ag disk diameters. The dashed line is the as-grown emission spectrum of the 330,240 and $130 \mathrm{~nm}$ samples.

The PL enhancement factor is shown in Fig. 3(b). The sample with Ag NDs of $330 \mathrm{~nm}$ diameter has the highest enhancement factor of about 2.7 at a wavelength of $523 \mathrm{~nm}$, while the as-grown emission peak wavelength is around $545 \mathrm{~nm}$. Considering the $380 \mathrm{~nm}$ sample, the peak enhancement factor is about 2.2 around $513 \mathrm{~nm}$. The $240 \mathrm{~nm}$ 
sample appears to have a very broad enhancement despite being below a factor of 1.5 , while at the same time the sample with the smallest diameter has the lowest enhancement factor.

However, when considering the LSP resonance through a reflectance measurement, as shown in Fig. 4(a), the $130 \mathrm{~nm}$ sample demonstrates the most pronounced resonance, although very weak as seen by the absorption spectra in Fig. 4(b). Absorption is obtained by $A=1-T-R$, where $T$ and $R$ are the transmittance and reflectance, respectively. From the absorption spectrum of the $240 \mathrm{~nm}$ sample, it would appear that an LSP resonance could be located in the long wavelength region. Thus we might understand how the enhancement factor can have such a flat shape as in Fig. 3(b), if we assume that the PL enhancement follows the LSP resonance.

Except for the $380 \mathrm{~nm}$ sample, the enhancement factors follow the trend of the absorption strengths. For instance, while the $130 \mathrm{~nm}$ sample has the smallest absorption it also has the smallest enhancement. The decrease in absorption is well correlated with the decrease in metal surface coverage as the diameters are decreased while the lattice periodicity remains constant. The reflectance spectra suggest that the increasing PL enhancement with metal surface coverage is not due to reflection. Measurement of the transmittance spectra includes both the specular and diffusive transmission using an integrating sphere. Therefore, the significant increase in the quantity $1-T-R$ is due to absorption as opposed to scattering, since the latter is already included in the quantity, $T$.

The observations show that decreasing the particle size while keeping the pitch fixed, results in a reduced fieldenhancement effect. This suggests that the dipole-dipole interaction is weakened. Similar effect has been observed for disordered nanoparticles, where the interaction is significantly reduced for pitch-to-size ratios larger than 2 [5]. Thus to keep the advantage of a periodic structure the Ag NDs must keep their close proximity, and the pitch should be reduced together with the particle size, keeping in mind that an optimum pitch exists for a given particle size [4].
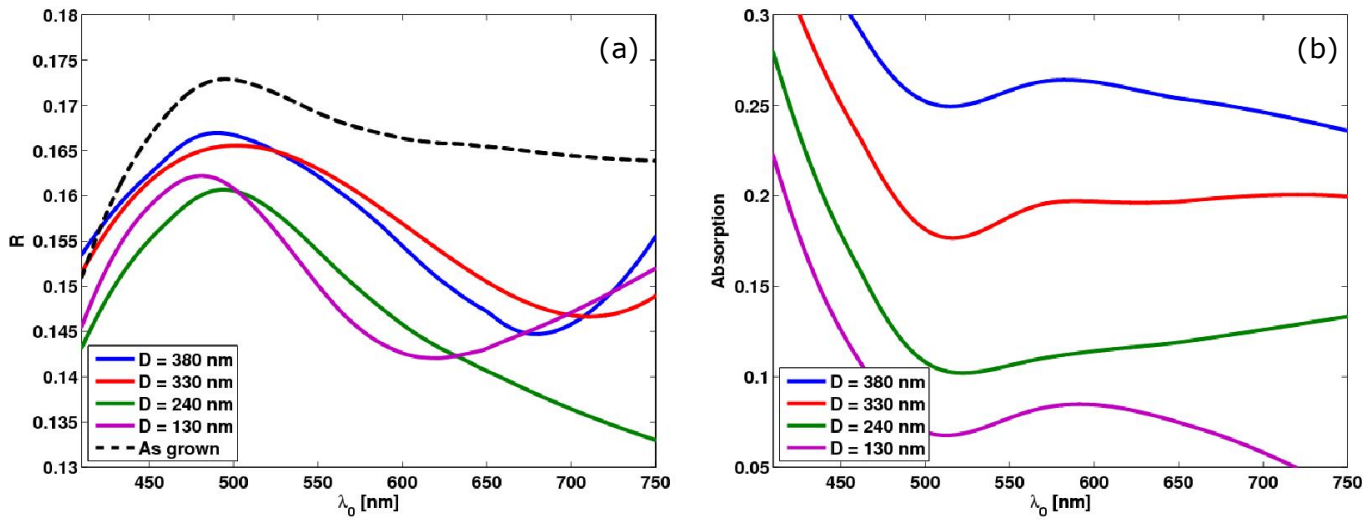

Figure 4: (a) Reflectance and (b) absorption spectra of the various diameters of Ag NDs.

\section{Conclusion}

A periodic pattern of disk-shaped Ag NPs were fabricated using NSL together with the Langmuir-Blodgett deposition technique of dielectric nanospheres serving as the lithography pattern template. The pitch and diameter of the final Ag NDs could be controlled by the initial and post-etch diameter of these nanospheres. PL characterizations revealed an enhancement by a factor of 2.7 for the Ag ND diameter of $330 \mathrm{~nm}$. Decreasing the Ag ND size below $240 \mathrm{~nm}$, while keeping the center-to-center distance at $600 \mathrm{~nm}$, has shown to be ineffective for PL enhancement. To obtain a smaller pitch, smaller dielectric nanospheres needs to be used as templates during the NSL process.

\section{References}

[1] K. Okamoto and T. Kawakami, "High-Efficiency InGaN/GaN Light Emitters Based on Nanophotonics and Plasmonics," IEEE Journal of Selected Topics in Quantum Electronics, 15, 1199 (2009).

[2] A. Neogi, C.-W. Lee, H. O. Everitt, T. Kuroda, A. Tackeuchi, and E. Yablonovitch, "Enhancement of spontaneous recombination rate in a quantum well by resonant surface plasmon coupling.” Phys. Rev. B, 66, 153305 (2002)

[3] D.-M. Yeh, C.-F. Huang, C.-Y. Chen, Y.-C. Lu, and C. C Yang, "Localized surface plasmon-induced emission enhancement of a green lightemitting diode," Nanotechnology, 19, 345201 (2008).

[4] B. Lamprecht, G. Schider , R. T. Lechner, H. Ditlbacher, J. R. Krenn, A. Leitner, and F. R. Aussenegg. "Metal nanoparticle gratings: Influence of dipolar particle interaction on the plasmon resonance," Phys. Rev. Lett. 84, 4721 (2000).

[5] W. Khunsin, B. Brian, J. Dorfmüller, M. Esslinger, R. Vogelgesang, C. Etrich, C. Rockstuhl, et al. (2011). "Long-distance indirect excitation of nanoplasmonic resonances," Nano letters, 11, 2765 (2011). 